\title{
Spatial distribution of environmental indicators in surface sediments of Lake Bolshoe Toko, Yakutia, Russia
}

Biskaborn et al.

\section{Supplementary Material}

\section{Multivariate statistics on diatom data}

Table a Results of DCA, PCA and RDA analysis. RDA is constrained to 5 significant variables.

\begin{tabular}{|c|c|c|c|c|c|}
\hline & Axis 1 & Axis 2 & Axis 3 & Axis 4 & \\
\hline \multicolumn{6}{|l|}{ DCA } \\
\hline Eigenvalues & 0.226 & 0.081 & 0.052 & 0.036 & \\
\hline Lengths of gradient & 2.069 & 1.487 & 0.996 & 1.019 & \\
\hline $\begin{array}{l}\text { Cumulative percentage variance of } \\
\text { species data }\end{array}$ & 16.4 & 22.2 & 26.0 & 28.6 & \\
\hline Sum of all eigenvalues & & & & & 1.381 \\
\hline \multicolumn{6}{|l|}{ PCA } \\
\hline Eigenvalues & 0.217 & 0.180 & 0.113 & 0.067 & : \\
\hline $\begin{array}{l}\text { Cumulative percentage variance of } \\
\text { species data }\end{array}$ & 21.7 & 39.6 & 51.0 & 57.6 & \\
\hline Sum of all eigenvalues & & & & & 1.000 \\
\hline \multicolumn{6}{|l|}{ RDA all variables } \\
\hline Eigenvalues & 0.217 & 0.180 & 0.113 & 0.067 & \\
\hline Taxon -environment correlations & 1.000 & 1.000 & 1.000 & 1.00 & \\
\hline Cumulative \% variance of taxon data & 21.7 & 39.6 & 51.0 & 57.6 & \\
\hline Of taxon-environment relation & 21.7 & 39.6 & 51.0 & 57.6 & \\
\hline Sum of all canonical eigenvalues & & & & & 1.000 \\
\hline \multicolumn{6}{|l|}{ RDA with 8 significant variables } \\
\hline Eigenvalues & 0.175 & 0.106 & 0.085 & 0.047 & \\
\hline Taxon -environment correlations & 0.918 & 0.833 & 0.863 & 0.951 & \\
\hline Cumulative $\%$ variance of taxon data & 17.5 & 28.1 & 36.6 & 41.3 & \\
\hline Of taxon-environment relation & 34.4 & 55.3 & 72.1 & 81.4 & \\
\hline Sum of all canonical eigenvalues & & & & & 0.507 \\
\hline
\end{tabular}


Table b Significance of variables as identified by manual forward selection in RDA and the variance they explain. Forward-selected variable was tested by a Monte Carlo permutation test (999 unrestricted permutation, $\mathrm{P} \leq 0.05$ )

\begin{tabular}{|c|c|c|c|}
\hline Variable & $\begin{array}{l}\text { Added with } \\
\text { selection }\end{array}$ & $\begin{array}{l}P \text { value } \\
\text { estimates }\end{array}$ & $F$ value \\
\hline Vegetation & 0.121 & 0.008 & 2.900 \\
\hline TOC/N & 0.08 & 0.016 & 2.032 \\
\hline TC & 0.07 & 0.020 & 1.892 \\
\hline EM 3 & 0.07 & 0.022 & 1.868 \\
\hline Sand & 0.05 & 0.030 & 1.457 \\
\hline WD & 0.046 & 0.031 & 2.196 \\
\hline Distance to the Shore & 0.04 & 0.034 & 1.907 \\
\hline Distance to the River & 0.03 & 0.046 & 1.774 \\
\hline $\begin{array}{l}\text { Total variance } \\
\text { explained by } \\
\text { significant variables }\end{array}$ & 0.507 & & \\
\hline SmecChlo & 0.048 & 0.106 & 1.55 \\
\hline Quarz & 0.036 & 0.230 & 1.238 \\
\hline Clay & 0.033 & 0.264 & 1.233 \\
\hline EM 1 & 0.030 & 0.402 & 1.052 \\
\hline KoalChlo & 0.026 & 0.690 & 0.769 \\
\hline Total variance & 0.680 & & \\
\hline
\end{tabular}

\section{Multivariate statistics on chironomid data}

Table c Results of DCA, PCA and RDA analysis. RDA is constrained to 5 significant variables.

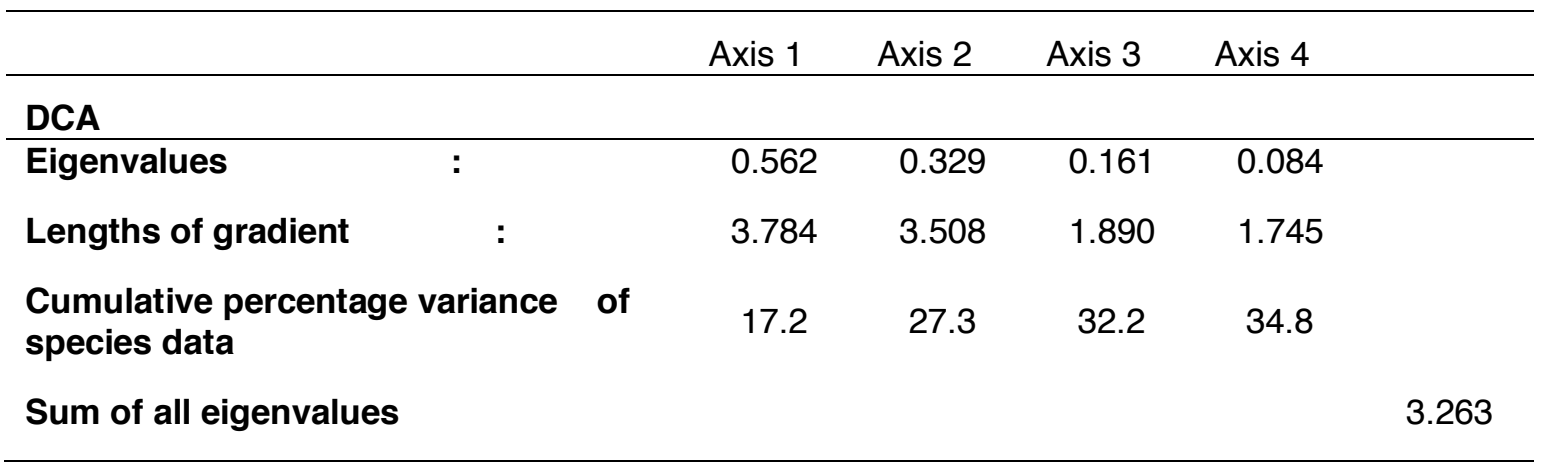




\begin{tabular}{|c|c|c|c|c|c|}
\hline PCA & & & & & \\
\hline Eigenvalues & 0.281 & 0.193 & 0.129 & 0.075 & \\
\hline $\begin{array}{l}\text { Cumulative percentage variance of } \\
\text { species data }\end{array}$ & 28.1 & 47.4 & 60.4 & 67.9 & \\
\hline Sum of all eigenvalues & & & & & 1.000 \\
\hline \multicolumn{6}{|l|}{ RDA all variables } \\
\hline Eigenvalues & 0.289 & 0.177 & 0.134 & 0.077 & \\
\hline Taxon -environment correlations & 1.000 & 1.000 & 1.000 & 1.000 & \\
\hline Cumulative $\%$ variance of taxon data & 28.9 & 46.7 & 60.1 & 67.8 & \\
\hline Of taxon-environment relation & 28.9 & 46.7 & 60.1 & 67.8 & \\
\hline Sum of all canonical eigenvalues & & & & & 1.000 \\
\hline \multicolumn{6}{|l|}{ RDA with 4 significant variables } \\
\hline Eigenvalues & 0.200 & 0.150 & 0.062 & 0.023 & \\
\hline Taxon -environment correlations & 0.881 & 0.932 & 0.895 & 0.683 & \\
\hline Cumulative $\%$ variance of taxon data & 20.0 & 35.0 & 41.2 & 43.4 & \\
\hline Of taxon-environment relation & 46.0 & 80.5 & 94.8 & 100.0 & \\
\hline Sum of all canonical eigenvalues & & & & & 0.435 \\
\hline
\end{tabular}


Table $\mathbf{d}$ Significance of variables as identified by manual forward selection in RDA and the variance they explain. Forward-selected variable was tested by a Monte Carlo permutation test (999 unrestricted permutation, $\mathrm{P} \leq 0.05$ )

\begin{tabular}{llll}
\hline Variable & $\begin{array}{l}\text { Added with } \\
\text { selection }\end{array}$ & $\begin{array}{l}\text { P value } \\
\text { estimates }\end{array}$ & F value \\
\hline TOC/N & 0.172 & 0.001 & 3.316 \\
WD & 0.106 & 0.031 & 2.196 \\
Distance to the River & 0.081 & 0.056 & 1.774 \\
Vegetation & 0.076 & 0.059 & 1.738 \\
Total variance & 0.435 & & \\
explained by & & & \\
significant variables & & & \\
TC & 0.053 & 0.249 & 1.243 \\
Distance to the Shore & 0.049 & 0.499 & 0.907 \\
Silt & 0.035 & 0.629 & 0.795 \\
Clay & 0.031 & 0.774 & 0.69 \\
Total variance & 0.603 & & \\
\hline
\end{tabular}

\section{End-member analysis of grain-size data}

Figure A End-member modelling results. Mean coefficient of determination $\mathrm{r}^{2}$ as function of grain size classes (left) and for samples (right).
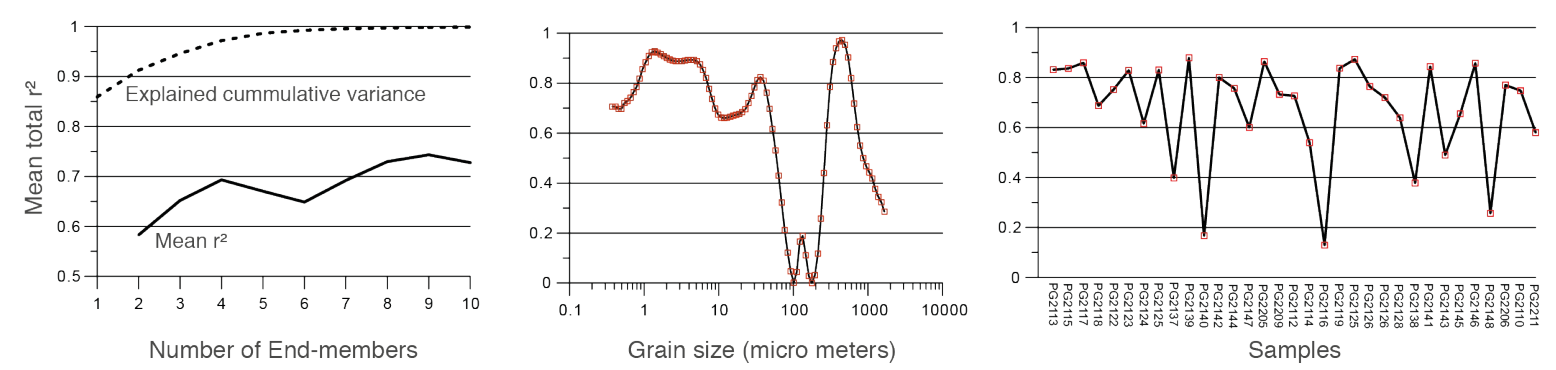